Tohoku Math. Journ.

Vol. 19, No. 4, 1967

\title{
ON THE MEAN OF AN ENTIRE FUNCTION AND THE MEAN OF THE PRODUCT OF TWO ENTIRE FUNCTIONS
}

\author{
T. V. LAKSHMINARASIMHAN
}

(Received January 20, 1967, revised May 8,1967)

Let $f(z)$ be an entire function, which is not a polynomial in general, of order $\rho$ and lower order $\lambda$. Let $I_{\delta}(r, f)$ be defined by

$$
I_{\delta}(r, f)=\left(\frac{1}{2 \pi} \int^{2 \pi}\left|f\left(r e^{i}\right)\right|^{\delta} d \theta\right)^{1 / \delta}, 0<\delta<\infty
$$

Then we have a theorem which was proved recently ([3], Theorem).

ThEOREM. Provided $\delta \geqq 1$ we have

$$
\limsup _{r \rightarrow \infty} \frac{\log \left[r I_{\delta}\left(r, f^{\prime}\right) / I_{\delta}(r, f)\right]}{\log r}=\rho, \quad 0 \leqq \rho \leqq \infty
$$

When $\rho$ is finite it would appear that (1) holds for $0<\delta<1$ as well, as the example $f(z)=\exp z$ would show.

Our main aim at present is to show that we are able to prove the following theorem in the case of functions of finite order when $0<\delta<1$.

THEOREM 1. When $f(z)$ is a function of finite order $\rho$ and $0<\delta<1$, then

$$
\limsup _{r \rightarrow \infty} \frac{\log \left[r I_{\delta}\left(r, f^{\prime}\right) / I_{\delta}(r, f)\right]}{\log r} \leqq \rho
$$

We need the following lemma for the proof.

LEMMA 1. If $0<\delta<1$, then for $r<R$,

$$
I_{\delta}\left(r, f^{\prime}\right)<C(\delta)(R-r)^{-1} I_{\delta}(R, f),
$$

where $C(\delta)$ is a constant depending on $\delta$ alone.

PROOF. It can be shown ([2], Lemma 1) that 
(3)

$$
I\left(r, f^{\prime}\right) \leqq(R-r)^{-1} I(R, f)
$$

where $I(t, u) \equiv I_{1}(t, u)$. Now let us assume that $f$ has no zeros in $|z|<R$. Choose $\psi=f^{\delta}$ so that

$$
\left|f^{\prime}\right|=\delta^{-1}|\psi|^{(1-\delta) / \delta}\left|\psi^{\prime}\right|, \quad \psi \equiv \psi\left(r \mathrm{e}^{i \theta}\right), \quad f \equiv f\left(r \mathrm{e}^{i \theta}\right)
$$

where the accents indicate the respective derivatives. Hence by Hölder's inequality

$$
\begin{aligned}
\int_{0}^{2 \pi}\left|f^{\prime}\right|^{\delta} d \theta & \leqq \delta^{-\delta} \int_{0}^{2 \pi}|\psi|^{1-\delta}\left|\psi^{\prime}\right|^{\delta} d \theta \\
& \leqq \delta^{-\delta}\left(\int_{0}^{2 \pi}|\psi| d \theta\right)^{1-\delta}\left(\int_{0}^{2 \pi}\left|\psi^{\prime}\right| d \theta\right)^{\delta}
\end{aligned}
$$

If we use (3) with $\psi$ in the place of $f$, we get from the above inequality,

$$
\begin{aligned}
\left(\int_{0}^{2 \pi}\left|f^{\prime}\right|^{\delta} d \theta\right)^{1 / \delta} & \leqq \delta^{-1}\left[\int_{0}^{2 \pi}\left|\psi\left(R e^{i \theta}\right)\right| d \theta\right]^{(1-\delta) / \delta} \times \int_{0}^{2 \pi}\left|\psi^{\prime}\right| d \theta \\
& \leqq \delta^{-1}\left[\int_{0}^{2 \pi}\left|\psi\left(R e^{i \theta}\right)\right| d \theta\right]^{(1-\delta) / \delta} \times(R-r)^{-1} \int_{0}^{2 \pi}\left|\psi\left(R e^{i \theta}\right)\right| d \theta \\
& \leqq \delta^{-1}(R-r)^{-1}(2 \pi)^{1 / \delta} I_{\delta}(R, f)
\end{aligned}
$$

which is equivalent to (2) when $C(\delta)=\delta^{-1}$. Next let us suppose that $f$ has zeros in $|z|<R$. Then it is known ([1], p.207) that $f(z)=f_{1}(z)+f_{2}(z)$, where $f_{1}$ and $f_{2}$ have no zeros in $|z|<R$ and $\left|f_{p}(z)\right|<2|f(z)|, p=1,2$. Hence we have from the previous result on using the familiar inequality that $|a+b|^{p}$ $\leqq|a|^{p}+|b|^{p}$ for $0 \leqq p \leqq 1$,

$$
\begin{aligned}
2 \pi\left[I_{\delta}\left(r, f^{\prime}\right)\right]^{\delta} & =\int^{2 \pi}\left|f^{\prime}\left(r e^{i \theta}\right)\right|^{\delta} d \theta \\
& \leqq \int_{0}^{2}\left|f^{\prime}{ }_{1}\left(r e^{i \theta}\right)\right|^{\delta} d \theta+\int^{2 \pi}\left|f_{2}^{\prime}\left(r e^{i \theta}\right)\right|^{\delta} d \theta \\
& \leqq[\delta(R-r)]^{-\delta}\left[\int_{0}^{2 \pi}\left|f_{1}\left(R e^{i \theta}\right)\right|^{\delta} d \theta+\int_{0}^{2 \pi}\left|f_{2}\left(R e^{i \theta}\right)\right|^{\delta} d \theta\right]
\end{aligned}
$$




$$
\leqq 2^{\delta+1}[\delta(R-r)]^{-\delta} \int_{0}^{2 \pi}\left|f\left(R e^{i \theta}\right)\right|^{\delta} d \theta
$$

Finally

$$
I_{\delta}\left(r, f^{\prime}\right) \leqq C(\delta)(R-r)^{-1} I_{\delta}(R, f)
$$

where $C(\delta)=2\left[2^{1 / \delta} / \delta\right]$.

PROOF OF THEOREM 1. Lemma 1 leads to the following inequality by a method which is available already ([3], pp.307-308).

$$
I_{\delta}\left(r, f^{\prime}\right) \leqq r^{\rho-1+\varepsilon} I_{\delta}(r, f), \quad 0<\delta<1, \quad r \geqq r_{0}(\varepsilon),
$$

where $\varepsilon$ is an arbitrarily small positive quantity.

The theorem follows from this inequality.

The above theorem also holds with $\lambda$, the lower order in the place of $\rho$ (Cf. [3], Lemma 4) and lim sup replaced by lim inf.

Let $f(z)$ and $g(z)$ be two entire functions and let $\alpha>0, \beta>0$,

$$
I_{\alpha, \beta}(r) \equiv I\left(r,|f|^{\alpha}|g|^{\beta}\right)=\left(\frac{1}{2 \pi} \int_{0}^{2 \pi}\left|f\left(r e^{i \theta}\right)\right|^{\alpha}\left|g\left(r e^{i \theta}\right)\right|^{\beta} d \theta\right)^{1 /(\alpha+\beta)}
$$

It is well known that $|f|^{\alpha}|g|^{\beta}$ is of class $P L\left([5]\right.$, p.9) and so $\log I_{\alpha, \beta}(r)$ is a convex function of $\log r$ ([5]). We will prove the following theorem which extends to two functions a result proved earlier for one function $f([6]$, Theorem 1).

THEOREM 2. If $f(z)$ and $g(z)$ are two functions, which are not polynomials, of orders $\rho_{f}$ and $\rho_{g}$ respectively, then

$$
(\alpha+\beta) \log I_{\alpha, \beta}(r) \sim \log \left[\max _{|z|=r}\left|f^{\alpha} g^{\beta}\right|\right], \quad r \rightarrow \infty,
$$

If $f=g$ and $\alpha=\beta=\gamma$ we get the result for one function $f$ as mentioned above.

For the proof we need the following lemma.

LEMMA 2. For the entire functions $f$ and $g$

$$
\rho=\lim _{r \rightarrow \infty} \sup _{r \rightarrow \infty} \frac{\log \log I_{\alpha, \beta}^{(r)}}{\log r} \leqq \rho_{f}+\rho_{g}\left(0 \leqq \rho_{f,}, \rho_{g} \leqq \infty\right) .
$$


PROOF. Since $|f|^{\alpha}|g|^{\beta}$ is of class $P L$, it follows that it is subharmonic in $|z| \leqq r<R$. Hence on $|z|=r$, we have by Poisson's formula for subharmonic functions

$$
I_{\alpha, \beta}(r) \leqq\left[\max _{|z|=r}\left|f^{\alpha} g^{\beta}\right|\right]^{1 /(\alpha+\beta)} \leqq[(R+r) /(R-r)]^{1 /(\alpha+\beta)} I_{\alpha, \beta}(R) .
$$

The lemma now follows from the left hand inequality in (4).

Proof of TheOREM 2. Since $\log I_{\alpha, \beta}(r)$ is a convex function of $\log r$

$$
\log I_{\alpha, \beta}(r)=\log I_{\alpha, \beta}\left(r_{0}\right)+\int_{r_{0}}^{r} \frac{m_{\alpha, \beta}(x)}{x} d x
$$

where $m_{\alpha, \beta}(x)$ is a non decreasing function of $x$. By Lemma 2, since $\rho_{f}+\rho_{g}<\infty$,

$$
\log I_{\alpha, \beta}(r)<r^{\rho+\varepsilon}, \quad r \geqq r_{0}(\varepsilon),
$$

where $\varepsilon$ is defined as before.

Also we get from (5) and (6)

$$
\int_{r}^{2 r} \frac{m_{\alpha, \beta}(x)}{x} d x<(2 r)^{\rho+\varepsilon}
$$

or

$$
(\log 2) m_{\alpha, \beta}(r)<(2 r)^{\rho+\varepsilon}
$$

and $\varepsilon$ being arbitrary

$$
m_{\alpha, \beta}(r)<r^{\rho+\varepsilon} .
$$

Hence for $r<R$

$$
\begin{aligned}
\int_{r}^{R} \frac{m_{\alpha, \beta}(x)}{x} d x & <m_{\alpha, \beta}(R) \log (R / r) \\
& =m_{\alpha, \beta}(R) \log \left(1+\frac{R-r}{r}\right) \\
& <R^{\rho+\varepsilon}[(R-r) / r] .
\end{aligned}
$$

Choosing $R$ such that 


$$
R=r\left[1+\frac{1}{r^{\rho+\varepsilon}}\right]
$$

we get

$$
\int_{r}^{R} \frac{m_{\alpha, \beta}(x)}{x} d x<2^{\rho+\varepsilon}
$$

This choice of $R$ is correct since in (5) we can take $r$ in the place of $r_{0}$ and $R>r$ in the place of $r$.

Hence from (4), (5), and (7)

$$
\begin{aligned}
&\left.\log _{|z|=r}\left|f^{\alpha} g^{\beta}\right|\right] \leqq \log \left(\frac{R+r}{R-r}\right)+(\alpha+\beta) \log I_{\alpha, \beta}(R) \\
&<\log \left(1+2 r^{\rho+\varepsilon}\right) \\
& \quad+(\alpha+\beta)\left[2^{\rho+\varepsilon}+\log I_{\alpha, \beta}(r)\right] \\
& \leqq(1+\varepsilon)(\alpha+\beta) \log I_{\alpha, \beta}(r)
\end{aligned}
$$

for all $r \geqq r_{0}(\varepsilon)$, $\varepsilon$ being defined as in previous cases. The theorem now follows from the inequalities (4) and (8).

Finally if we define the new mean $I_{\alpha, \beta}^{(k)}(r)$ by

$$
I_{\alpha, \beta}^{(k)}=r^{-k-1} \int_{0}^{r} x^{k} I_{\alpha, \beta}(x) d x, \quad k+1>0
$$

the following theorem can be proved.

THEOREM 3. If $\rho_{f}$ and $\rho_{g}$ are finite,

$$
\limsup _{r \rightarrow \infty}\left[I_{\alpha, \beta}(r) / I_{\alpha, \beta}^{(\kappa)}(r)\right]^{1 / \log r} \leqq e^{\rho_{f}+\rho_{a}} .
$$

The proof depends on Lemma 2 and the fact that $\log I_{\alpha, \beta}^{(\kappa)}(r)$ is a convex function of $\log r$ (Cf.[4],pp.1277-79). We omit this for conciseness.

Our thanks are due to the referee for his useful comments.

ADDED IN PROOF. The author wishes to express his thanks to Professor W.K. Hayman who pointed out an error in the original form of Theorem 2. 


\section{REFERENCES}

[1] G.H.HARDy AND J. E. Littlewood, Some properties of Fourier constants, Math. Ann. Ser. 2, 97(1927), 159-209.

[2] T.V.LAKSHMINARASIMHAN, On the mean value of the modulus of an entire function and its derivatives, Quart. Journ. Math. (Oxford) Ser.2, 14(1963), 16-20.

[3] T.V.LAKSHMINARASIMHAN, On a theorem concerning the means of an entire function and its derivative, Journ. London Math. Soc., 40(1965), 305-308.

[4] T.V.lakshminarasimhan, A note on means of entire functions, Proc. Amer. Math. Soc., 16(1965), 1277-1279.

[5] T. RADO, Subharmonic functions, Chelsea, New York, 1949.

[6] Q.I.Rahman, On means of entire functions, Proc. Amer. Math. Soc., 9(1958), 748-750.

DEPARTMENT OF MATHEMATICS

MADRAS CHRISTIAN COLLEGE

MADRAS-59, INDIA. 